\title{
The double burden of age and disease on cognition and quality of life in bipolar disorder
}

Sara L. Weisenbach ${ }^{1,2}$, David Marshall ${ }^{1}$, Anne L. Weldon ${ }^{3}$, Kelly A. Ryan ${ }^{1}$, Aaron C. Vederman ${ }^{1}$, Masoud Kamali ${ }^{1}$, Jon-Kar Zubieta ${ }^{1}$, Melvin G. McInnis ${ }^{1}$ and Scott A. Langenecker ${ }^{1,3}$

\footnotetext{
${ }^{1}$ University of Michigan Medical School, Department of Psychiatry, Ann Arbor, MI, USA

${ }^{2}$ Jesse Brown Veterans Affairs Medical Center, Chicago, IL, USA

${ }^{3}$ University of Illinois at Chicago, Department of Psychiatry, Chicago, IL, USA

Correspondence to: S. L. Weisenbach, PhD, E-mail: sweisenbach@psych.uic.edu
}

Objective: Bipolar disorder (BPD) and normal aging are known to impact cognitive skills and health-related quality of life (HRQOL). This study investigated how aging and disease interact in predicting cognitive and psychosocial outcomes.

Methods: Eight cognitive and ten subjective HRQOL domain ratings were measured. Subjects included 80 young (18-29 years) and late middle-aged (50-65 years) BPD patients in the euthymic phase and 70 age-equivalent healthy comparison participants.

Results: An age X disease interaction was detected in three domains of cognitive functioning that reflect emotion processing, processing speed, and executive functioning skills, with BPD patients in the older group performing most poorly. There was a double burden of aging and disease on reported ability to perform physical tasks. However, regardless of age, disease status was associated with lower ratings of HRQOL in the psychosocial/affective sphere and the majority of cognitive domains. Post hoc analyses revealed that number of years ill was positively associated with select HRQOL ratings in older, but not younger BPD adults.

Conclusions: These findings may stimulate future longitudinal study of cognition and quality of life in BPD patients across the life span, focusing on additive and interactive effects of aging and disease burden, which could culminate in developing more effective treatment and rehabilitation strategies for this traditionally challenging to treat population. Copyright (C) 2014 John Wiley \& Sons, Ltd.

Key words: bipolar disorder; cognition; quality of life; aging

History: Received 29 July 2013; Accepted 06 January 2014; Published online 18 February 2014 in Wiley Online Library (wileyonlinelibrary.com)

DOI: 10.1002 /gps.4084

\section{Introduction}

\section{Bipolar disorder, aging, and cognition}

Deficits in psychomotor speed, attention, executive functions, memory, and fine motor skills have been widely reported in bipolar disorder (BPD), both during active states of mania and depression and during the euthymic state (van Gorp et al., 1998; Rubinsztein et al., 2000; Zubieta et al., 2001; Altshuler et al., 2004; Martinez-Aran et al., 2004; Burdick et al., 2006; Bora et al., 2009; Langenecker et al., 2010). Normal aging is also known to impact a number of cognitive skills
(Villardita et al., 1985), although how aging and disease interact in the context of BPD is unclear. McKinney and Sibille (2013) propose that age-related genetic functional changes may promote vulnerability to develop diseases, such as depression, through alterations in gene expression for genes that are disease-promoting. A similar model might be applied to the study of behavioral consequences of early-onset mood disorders in the context of aging. Specifically, age-related brain changes may promote vulnerability to develop cognitive and functional problems, which may be most evident in domains for which decrement is associated with mood disorder. Alternatively, or in addition to, change in 
domains that are impacted even early in the disease process may demonstrate accelerated decline during aging, as a result of increased vulnerability. To more clearly explicate potential interactive and noninteractive effects of age-disease effects in mood disorder, we propose a testable model that describes the trajectory of cognition and function from young to later life BPD (Figure 1).

There is a growing volume of literature concerning cognition in middle aged and older adults with BPD. Case-control studies of older euthymic BPD patients find poorer performance in multiple cognitive domains, including processing speed, language, visuomotor skills, working memory, verbal fluency, episodic memory, executive functioning, and psychomotor speed (Delaloye et al., 2009; Schouws et al., 2009; Gildengers et al., 2012). Results from longitudinal studies of cognition in middle-aged/older adults with BPD are variable, depending upon the time frame studied. At two-year follow-up, no differences in the trajectory of cognitive changes were found, both relative to healthy, same-age comparisons (Delaloye et al., 2011) nor when comparing symptomatic to asymptomatic patients (Braw et al., 2013). Another study conducted over 3 years, however, found that BPD patients demonstrated more variability in performance on a composite of global cognition, relative to the healthy comparison group (Depp et al., 2008). When the assessment period was lengthened to 9 years, young and middle-aged euthymic BPD patients exhibited declined executive functioning, slight improvement in attention, and no changes in verbal learning and memory relative to healthy, age-matched comparison groups (Torrent et al., 2012).

Although the aforementioned studies have elucidated some of the cognitive problems that middle aged and

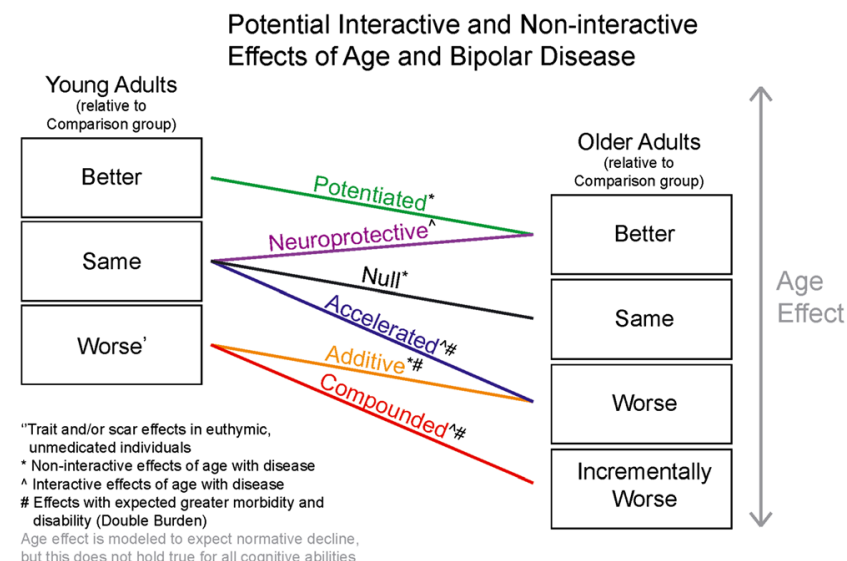

Figure 1 Potential interactive and non-interactive effects of age and bipolar disease. older patients with BPD experience, only two known studies have included both younger and older samples to assess the interaction of aging and disease. The first study included both patients with BPD and unipolar depression in the same group, and presumably, in both active and euthymic/remitted states (though this is not explicitly stated; Gualtieri and Johnson, 2008). Participants were divided into five age groups from ages 18 to 86 years. Patients performed less well than sameage comparison groups across all cognitive domains assessed, and decline was shown to accelerate in patients with mood disorders after the age of 65 years in every domain except complex attention. In the second study, 71 euthymic BPD patients and 82 healthy comparison participants, ranging in age from 18 to 70, underwent a short battery of cognitive tests. When used as a covariate, age did not have any differential impact on performance for tests measuring memory and executive functioning (Sarnicola et al., 2009). These studies are limited, in that they either included individuals in both the active and euthymic states (in the case of the first study), or did not study cognition comprehensively (in the case of the second study).

\section{Quality of life in bipolar disorder}

While there has been a great deal of attention paid to cognition in the context of aging and disease, less emphasis has been placed on subjective quality of life, which, although related to cognitive skills, should be considered separately for the purpose of treatment outcomes. It is well documented that individuals with BPD report a lower quality of life than their healthy counterparts, both in the realms of physical and mental health (Michalak et al., 2005; Bonnin et al., 2012). Further, with normal aging comes greater variability in quality of life, given higher rates of physical disease burden and disability than during younger ages. It is not clear, however, how aging and disease interact with subjective health-related quality of life (HRQOL), which is highly relevant to treatment providers caring for older adults with BPD.

\section{Aims of the current study}

This study was implemented to address methodological and power weaknesses in the literature that have precluded the field from understanding critical disease and age-related issues and interactions. It sought to explicate how aging and disease interact in impacting cognitive function and HRQOL ratings among 
younger and older middle-aged BPD patients in the euthymic phase of illness relative to age-equivalent non-BPD peers. Consistent with previous literature on cognitive functioning in normal aging and in $\mathrm{BPD}$, we expected age and BPD to interact, such that older middle-aged adults with BPD would perform most poorly on measures of cognitive functioning that are particularly sensitive to the effects of disease and aging, including executive functioning, memory, and processing speed. In the model proposed, this would be observed as an accelerated or compounded effect of disease with aging. We predicted an interaction of age and disease on HQROL ratings, such that the older group with BPD would be especially prone to report lower quality of life ratings, both in physical and emotional domains, such that a compounded effect of disease with aging is observed.

\section{Methods}

\section{Participants}

Study participants were recruited for the Heinz C. Prechter Longitudinal Bipolar Study, a study of phenotypic and biological outcomes of BPD at the University of Michigan from October 2005 to December 2010 (Langenecker et al., 2010). Recruitment methods and inclusion/exclusion criteria have been reported previously (Langenecker et al., 2010) and are included in supplemental material. Of the 586 participants recruited for the longitudinal cohort, 80 individuals with confirmed BPD I $(n=70)$ or BPD II $(n=10)$ in the euthymic state and 78 healthy comparison (HC) subjects were included in the present study. We specifically selected individuals younger than 30 years and ages 50 to 65 years for inclusion in the current comparison study, although the larger longitudinal cohort includes individuals aged 18 to
65 years, as well as patients with active state BPD. The BPD and HC groups extracted from the larger data set were matched for age, then education, then sex, resulting in the exclusion of nine BPD patients and 33 HC. More specifically, matches for BPD patients were derived from the HC group for age within 2 years, then education within 2 years, and finally sex. Of note, there are three more males in the $\mathrm{HC}$ group than the BPD group and five more females in the BPD group relative to the $\mathrm{HC}$ group.

This study was approved by the Institutional Review Board at the University of Michigan, and all participants gave informed consent prior to participation. The sample was divided into two age groups: those between the ages of 18 and 29 years $(n=95)$ and those aged 50 to 65 years $(n=63)$. Within each of the younger and older groups, there were no significant differences between the BPD and HC groups on age, education, or sex (Table 1).

\section{Clinical variables}

Clinical variables were extracted from the baseline Diagnostic Interview for Genetic Studies (Nurnberger et al., 1994), including medication loading (Hassel et al., 2008) and years of illness. Table 1 characterizes the young and older BPD groups on all clinical variables.

\section{Measures}

All participants received a research-defined comprehensive neuropsychological evaluation that focused on areas known to be adversely impacted in $\mathrm{BPD}$, including memory, attention and executive functioning, psychomotor speed, and emotion processing (Langenecker et al., 2010) and completed a self-report measure of HRQOL, the Short Form 36 Health Survey (SF-36; McHorney et al., 1993,

Table 1 Sample demographic and clinical characteristics*

\begin{tabular}{|c|c|c|c|c|c|c|c|}
\hline & Sex & Age & Educ. & HAM-D & YMRS & Years III" & MedBur \\
\hline Young HC & $20 \mathrm{M} 28 \mathrm{~F}$ & $24.3 \pm 3.2$ & $15.8 \pm 1.9$ & $1.2 \pm 1.6$ & $0.2 \pm 0.5$ & - & - \\
\hline Young BPD & $20 \mathrm{M} 27 \mathrm{~F}$ & $24.1 \pm 3.1$ & $15.1 \pm 1.8$ & $3.2 \pm 3.1$ & $1.0 \pm 1.7$ & $7.8 \pm 4.7$ & $1.3 \pm 1.9$ \\
\hline Older HC & $17 \mathrm{M} 13 \mathrm{~F}$ & $56.9 \pm 4.0$ & $15.6 \pm 2.3$ & $0.2 \pm 0.3$ & $0.3 \pm 0.6$ & - & - \\
\hline Older BPD & $14 \mathrm{M} 19 \mathrm{~F}$ & $56.4 \pm 4.7$ & $16.5 \pm 2.1$ & $2.8 \pm 2.6$ & $0.8 \pm 1.3$ & $35.8 \pm 7.8$ & $1.4 \pm 1.7$ \\
\hline
\end{tabular}

HC, healthy control; BPD, bipolar disorder; M, male; F, female; Educ., education; HAM-D, Hamilton Depression Rating Scale (Hamilton, 1967); YMRS, Young Mania Rating Scale (Young et al., 1978); MedBur, medication burden.

The majority of participants classified themselves as Caucasian (64 BPD, $58 \mathrm{HC}$ ). The sample also consisted of individuals classifying themselves as Black or African American (5 BPD, 8 HC), Asian (4 BPD, 7 HC), American Indian/Alaskan Native (1 HC), and More Than One Race (4 BPD, 4 HC). Race was not reported for two BPD participants. Refer to Table 1 for a complete characterization of sample demographics.

- Older individuals with BPD reported greater years of illness relative to younger individuals with $\mathrm{BPD}, t(77)=-18.40, p<0.001$. 
1994; Ware and Sherbourne, 1993). Standard data reduction techniques were utilized using conceptually and theoretically categorized variables (see Langenecker et al., 2010 for a full description). Truncation was carried out separately for BPD and $\mathrm{HC}$ to avoid biases of misapplication from one group to another. Supplemental Table 1 lists the measures included in each cognitive domain and the internal consistency value for this particular sample for factors with three or more variables.

\section{Statistical analyses}

The alpha level was set at $p<0.05$ for all analyses using SPSS 19.0. Independent $t$-tests and chi-square analyses were performed to test between-group differences in demographic variables. Multivariate analyses (MANOVA) were completed to examine the effects of age (young versus middle aged) and diagnosis (BPD versus comparison), and the interaction of age and diagnosis on the eight cognitive factor scores and subscales of the SF-36, respectively. Education was entered as a covariate for the MANOVA for cognitive factors, as education is associated with cognitive reserve (Liu et al., 2013) and may obscure age by disease interactions. A series of post hoc exploratory multiple regression analyses were computed to investigate the impact of clinical variables on cognitive performance and HRQOL ratings in the BPD group. We were interested in evaluating independent contributions of age and duration of illness; however, because they were so highly correlated $(r=0.93)$, this disallowed for independent evaluations. As a result, we elected to perform regression analyses separately for the younger and middle-aged groups. To further prevent against confounding years of illness with age, the years of illness variable was normalized to a $\mathrm{z}$ distribution within each age group. In the first step of each regression, education (for cognitive variables only) and symptom severity ratings (Hamilton Depression Rating Scale and Young Mania Rating Scale scores) were entered, and in the second step, medication load and normalized years of illness were entered. Data for years of illness was missing for one subject. Because participants in the BPD group were taking a number of medications that varied in class and dose that could impact cognitive functioning, we quantified medication load by using criteria based on prior literature (Hassel et al., 2008; Almeida et al., 2009; see supplemental material for complete description). Medication load was missing for five participants.

\section{Results}

\section{Group comparisons for cognitive performance}

The main effects of age group, $F(8,124)=12.57$, $p<0.001$, and diagnosis, $F(8,124)=4.59, p<0.001$ were significant in the omnibus MANCOVA analysis. The interaction of age group by diagnosis was not significant, $F(8,124)=1.07, p=0.39$. Education was a significant covariate, $F(8,124)=2.95, p<0.01$. Post hoc analysis of covariances revealed main effects of age group for all eight cognitive factors. Main effects of diagnosis were detected for fine motor, visual memory, emotion processing, verbal fluency and processing speed (VFPS), and processing speed with interference resolution (PSIR). A significant interaction of age group by diagnosis was found for emotion processing, VFPS, and PSIR, (Table 2 and Figure 2). Follow-up analysis of covariances were conducted for these three factors to determine the specific nature of the interaction (Table 3). In the younger group, individuals with BPD performed more poorly than comparisons only in the domain of PSIR. Younger groups were equivalent for emotion processing and VFPS. In contrast, in the middle-aged group, BPD patients performed significantly more poorly than comparisons in PSIR and VFPS, with marginally significant differences detected for emotion processing. Thus, in the proposed model, PSIR represents a compounded effect of disease in the context of aging, whereas VFPS and emotion processing represent an accelerated effect of disease in the context of aging.

\section{Group comparisons for quality of life}

The main effects of age group, $F(8,119)=5.65$, $p<0.001$, and diagnosis, $F(8,119)=8.89, p<0.001$, and the interaction of age group $\mathrm{X}$ diagnosis, $F(8$, $119)=2.24, p<0.05$, were significant in the omnibus MANOVA analysis. Post hoc analysis of variances revealed main effects of age for physical functioning, role-physical, bodily pain, and the physical health component score. The main effect of diagnosis was present for all eight domains of functioning and the physical health and mental health component scores. A significant interaction of age group $\mathrm{X}$ diagnosis was detected for role-physical (Table 4, Figure 3). Follow-up $t$-tests were conducted for this factor to determine the specific nature of the interaction (Table 3). Although both younger and middle-aged BPD participants rated themselves as having lower quality of life than their comparison peers, the 
Table 2 Cognitive functioning across eight domains by age and disease status, covarying for education

\begin{tabular}{|c|c|c|c|}
\hline Cognitive measure & Source & $F$ & $p$ \\
\hline \multirow[t]{4}{*}{ Fine motor dexterity } & Education & 2.63 & 0.107 \\
\hline & Age group & 41.19 & $<0.001$ \\
\hline & BPD status & 15.36 & $<0.001$ \\
\hline & Age group X BPD status & 1.73 & 0.190 \\
\hline \multirow[t]{4}{*}{ Auditory memory } & Education & 9.13 & 0.003 \\
\hline & Age group & 6.04 & 0.015 \\
\hline & BPD status & 0.00 & 0.984 \\
\hline & Age group X BPD status & 0.03 & 0.863 \\
\hline \multirow[t]{4}{*}{ Visual memory } & Education & 0.03 & 0.858 \\
\hline & Age group & 20.76 & $<0.001$ \\
\hline & BPD status & 6.56 & 0.012 \\
\hline & Age group X BPD status & 1.28 & 0.260 \\
\hline \multirow[t]{4}{*}{ Emotion processing } & Education & 5.23 & 0.024 \\
\hline & Age group & 75.56 & $<0.001$ \\
\hline & BPD status & 5.11 & 0.025 \\
\hline & Age group X BPD status & 5.18 & 0.024 \\
\hline Verbal fluency and & Education & 11.81 & 0.001 \\
\hline \multirow[t]{3}{*}{ processing speed } & Age group & 16.97 & $<0.001$ \\
\hline & BPD status & 10.23 & 0.002 \\
\hline & Age group X BPD status & 3.97 & 0.048 \\
\hline Conceptual reasoning & Education & 11.21 & 0.001 \\
\hline Cognitive measure & Source & $F$ & $p$ \\
\hline \multirow[t]{3}{*}{ and set shifting } & Age group & 12.32 & 0.001 \\
\hline & BPD status & 1.02 & 0.315 \\
\hline & Age group X BPD status & 1.39 & 0.240 \\
\hline Processing speed with & Education & 5.76 & 0.018 \\
\hline \multirow[t]{3}{*}{ interference resolution } & Age group & 46.13 & $<0.001$ \\
\hline & BPD status & 28.20 & $<0.001$ \\
\hline & Age group X BPD status & 4.94 & 0.028 \\
\hline \multirow{4}{*}{ Inhibitory control } & Education & 1.49 & 0.224 \\
\hline & Age group & 5.65 & 0.019 \\
\hline & BPD status & 3.39 & 0.068 \\
\hline & Age group X BPD status & 0.06 & 0.804 \\
\hline
\end{tabular}

BPD, bipolar disorder.

$d f=(1131)$ for all variables.

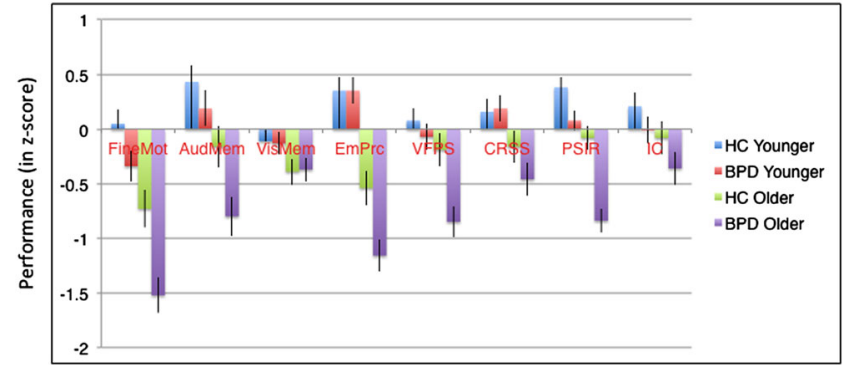

Figure 2 Disease by age on cognitive functioning. The main effect of age is significant for all eight cognitive factors. The main effect of diagnosis is significant for fine motor dexterity, visual memory, emotion processing, verbal fluency and processing speed, and processing speed with interference resolution. A significant interaction of age by diagnosis is present for emotion processing, verbal fluency and processing speed, and processing speed with interference resolution (all $p s<0.05$ ). Performance values are in z scores, which have a mean of 0 and $\mathrm{SD}$ of 1 . Note: HC, healthy control; BPD, bipolar disorder; FineMot, fine motor dexterity; AudMem, auditory memory; VisMem, visual memory; EmPrc, emotion processing; VFPS, verbal fluency and processing speed; CRSS, conceptual reasoning and set shifting; PSIR, processing speed with interference resolution; IC, inhibitory control. difference was larger in the older group. Thus, in the proposed model, functioning with regard to physical role would represent a compounded effect of disease in the context of aging.

Relationships of cognitive performance to clinical variables in BPD patients

After accounting for the impact of education (cognitive outcomes only) and symptom severity, years of illness positively predicted Role-Emotional $(t=2.48, p=0.02)$ and the Mental Component Scale $(t=2.32, p=0.03)$ on the SF-36 in the older group only. Years of illness predicted none of the HRQOL ratings in the younger group and did not predict cognitive performance in either group. Medication burden predicted social function $(t=-2.15, p=0.04)$ in the younger group. 
Table 3 Post hoc tests for significant age by disease interactions

\begin{tabular}{|c|c|c|c|c|c|}
\hline Age group & Cognitive domain & Source & $d f$ & $F / t$ & $p$ \\
\hline \multicolumn{6}{|l|}{ Young } \\
\hline & \multirow[t]{3}{*}{ Emotion processing } & Education & 1 & 7.74 & 0.007 \\
\hline & & BPD status & 1 & 0.16 & 0.694 \\
\hline & & Error & 85 & & \\
\hline & \multirow[t]{3}{*}{ Verbal fluency with proc speed } & Education & 1 & 3.58 & 0.062 \\
\hline & & BPD status & 1 & 1.66 & 0.201 \\
\hline & & Error & 88 & & \\
\hline & \multirow{3}{*}{ Proc speed with interference resolution } & Education & 1 & 1.71 & 0.195 \\
\hline & & BPD status & 1 & 8.7 & 0.004 \\
\hline & & Error & 85 & & \\
\hline & \multirow[t]{2}{*}{ Role-physical } & BPD status & 1 & & 0.038 \\
\hline & & Error & 76 & 5.71 & $<0.001$ \\
\hline \multirow{11}{*}{ Middle-aged } & \multirow[t]{3}{*}{ Emotion processing } & Education & 1 & 2.41 & 0.126 \\
\hline & & BPD status & 1 & 3.35 & 0.072 \\
\hline & & Error & 56 & & \\
\hline & \multirow{3}{*}{ Verbal fluency with proc speed } & Education & 1 & 8.51 & 0.005 \\
\hline & & BPD status & 1 & 11.7 & 0.001 \\
\hline & & Error & 58 & & \\
\hline & \multirow{3}{*}{ Proc speed with interference resolution } & Education & 1 & 3.85 & 0.055 \\
\hline & & BPD status & 1 & 20.67 & $<0.001$ \\
\hline & & Error & 55 & & \\
\hline & \multirow[t]{2}{*}{ Role-physical } & BPD status & 1 & 3.01 & 0.004 \\
\hline & & Error & 52 & & \\
\hline
\end{tabular}

BPD, bipolar disorder.

\section{Discussion}

This study considers the double burden of aging and disease status on cognition and HRQOL in the context of BPD. Late middle-aged euthymic adults with BPD demonstrate particularly poorer performance in the domains of emotion processing, processing speed, and aspects of executive functioning (i.e., verbal fluency, attentional shifting, and interference resolution). PSIR demonstrated a compounded effect with aging in the context of BPD, while VFPS and emotion processing characterized an accelerated effect of aging in BPD. At the same time, verbal memory and other aspects of executive functioning, such as inhibitory control, conceptual reasoning, and set shifting were impacted only by age, regardless of disease status. Both aging and disease impacted fine motor skills and visual memory skills, albeit independently. Results are consistent with past work identifying worsened executive functioning skills and slower processing speed in middle aged and older adults with BPD relative to their non-BPD peers (Delaloye et al., 2009, 2011; Schouws et al., 2009; Gildengers et al., 2012; Braw et al., 2013). A meta-analysis of cognitive dysfunction in euthymic BPD identified moderate to large effect sizes for executive functioning, processing speed, in addition to memory in euthymic BPD (Torres et al., 2007). Thus, when the effects of aging are compounded with potentially long standing pathophysiology in areas of the brain underlying these skills, it is not surprising to find especially poor performance in these domains among older people with BPD.

Findings are in contrast to previous work finding verbal memory deficits in aging BPD patients relative to same-age healthy comparisons (Delaloye et al., 2009; Schouws et al., 2009; Gildengers et al., 2012). It is likely that the age of our older sample (50-65 years) explains the discrepancy in findings, as the aforementioned studies included older participants than the current sample. Participants in our sample were also highly educated, and thus likely presented with more cognitive reserve than participants in the majority of studies. Further, Schouws and colleagues (2009) also included patients with late onset BPD, whereas we limited our sample to those with early-onset BPD. It is possible that processing speed and executive functioning decline at an earlier age than do memory skills in middle aged and older adults with $\mathrm{BPD}$, though this conjecture would need to be confirmed with a longitudinal study. Certainly, findings of declined executive functioning, but not memory, among BPD patients over 9 years (Torrent et al., 2012) would lend partial support to this possibility.

Affective processing deficits have been studied less often, relative to other cognitive skills, in middle-aged and older patients with BPD, though our cross-sectional results suggest that they may decline at an accelerated 
Table 4 Health-related quality of life ratings by age and disease status

\begin{tabular}{|c|c|c|c|}
\hline HRQOL subscale & Source & $F$ & $p$ \\
\hline \multirow[t]{3}{*}{ Physical functioning } & Age group & 16.53 & $<0.0001$ \\
\hline & BPD status & 24.40 & $<0.0001$ \\
\hline & Age group X BPD status & 1.83 & 0.178 \\
\hline \multirow[t]{3}{*}{ Role-physical } & Age group & 5.35 & 0.022 \\
\hline & BPD status & 25.23 & $<0.0001$ \\
\hline & Age group X BPD status & 6.30 & 0.013 \\
\hline \multirow[t]{3}{*}{ Bodily pain } & Age group & 4.53 & 0.035 \\
\hline & BPD status & 11.37 & 0.001 \\
\hline & Age group X BPD status & 0.21 & 0.647 \\
\hline \multirow[t]{3}{*}{ General health } & Age group & 0.46 & 0.501 \\
\hline & BPD status & 26.76 & $<0.0001$ \\
\hline & Age group $X$ BPD status & 0.75 & 0.387 \\
\hline \multirow[t]{3}{*}{ Physical health component } & Age group & 12.87 & $<0.0001$ \\
\hline & BPD status & 10.49 & 0.002 \\
\hline & Age group X BPD status & 3.02 & 0.085 \\
\hline \multirow[t]{3}{*}{ Vitality } & Age group & 3.39 & 0.068 \\
\hline & BPD status & 51.66 & $<0.0001$ \\
\hline & Age group X BPD status & 1.76 & 0.187 \\
\hline \multirow[t]{3}{*}{ Social functioning } & Age group & 0.04 & 0.849 \\
\hline & BPD status & 51.79 & $<0.0001$ \\
\hline & Age group X BPD status & 0.02 & 0.895 \\
\hline HRQOL subscale & Source & $F$ & $p$ \\
\hline \multirow{3}{*}{ Role-emotional } & Age group & 0.98 & 0.324 \\
\hline & BPD status & 36.92 & $<0.0001$ \\
\hline & Age group X BPD status & 1.30 & 0.256 \\
\hline \multirow[t]{3}{*}{ Mental health } & Age group & 0.14 & 0.708 \\
\hline & BPD status & 51.84 & $<0.0001$ \\
\hline & Age group X BPD status & 0.16 & 0.693 \\
\hline \multirow[t]{3}{*}{ Mental health component } & Age group & 3.89 & 0.051 \\
\hline & BPD status & 54.73 & $<0.0001$ \\
\hline & Age Group X BPD status & 1.83 & 0.179 \\
\hline
\end{tabular}

BPD, bipolar disorder; HRQOL, health-related quality of life. $\mathrm{df}=(1126)$ for all variables.

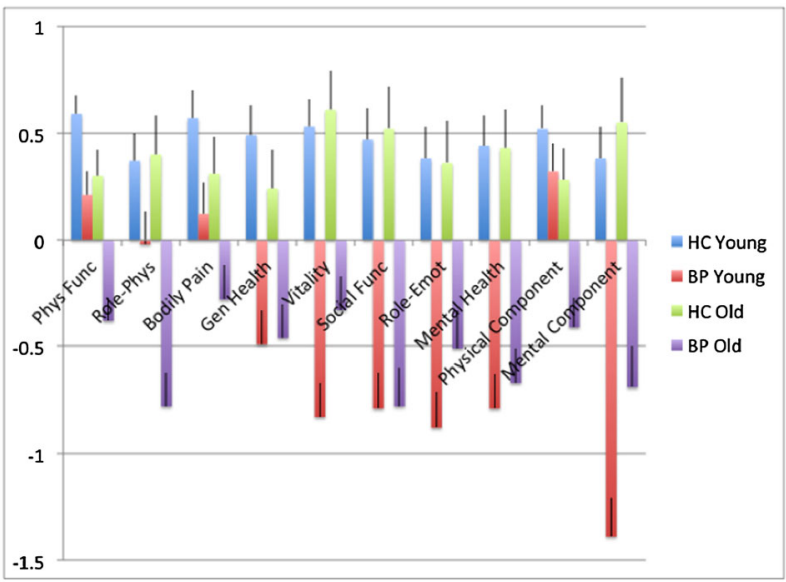

Figure 3 Disease by age on self-reported physical and emotional functioning. Significant effects of age are present for physical functioning, role-physical, bodily pain, and the physical health component score (not depicted). The main effect of diagnosis is found for all eight domains of functioning and the Physical and Mental Health component scores (not depicted). A significant interaction of age $\mathrm{X}$ diagnosis is detected for role-physical (all $p \mathrm{~s}$ 0.05). Performance values are in $\mathrm{z}$ scores, which have a mean of 0 and SD of 1. Note: HC, healthy control; BPD, bipolar disorder; Phys Func, physical functioning; Role Phys, role-physical; Gen Health, general health; Social Func, social functioning; Role Emot, role-emotional. pace with age. Interestingly, a longitudinal structural imaging study of 20 adults with BPD demonstrated larger declines in hippocampus and fusiform gyrus over 4 years as compared with same-age comparisons (Moorhead et al., 2007). These regions are important to emotion processing, and such neurodegenerative changes may help explain why the older patients with BPD performed particularly poorly on measures of emotion processing.

Our results also suggest that there is a compounded effect of aging and disease in the reported ability of a person to perform physical tasks, and aging negatively contributes to nearly all aspects of physical health, though it is a disease status, regardless of age group, that adversely impacts emotional health characteristics, such as having a sense of vitality and the ability to engage in social interactions. Thus, while select cognitive skills and engagement in physical activities may demonstrate accelerated changes with aging in the context of $\mathrm{BPD}$, emotional health ratings are not augmented with aging in BPD. Rather, emotional health is uniformly lower in both younger and older 
patient groups, relative to the comparison group. In the younger BPD group only, medication burden was related to poorer ratings in the domain of Social Function, perhaps suggesting that disease burden and/or medication side effects impact a person's ability to engage in social relationships to a degree that is satisfying. It is important for caregivers working with aging BPD patients to identify physical limitations and work toward assisting patients in identifying solutions, particularly given the difficulties that some of these patients have with executive functioning that can impair planning and problem-solving. Clinicians working with BPD patients of all ages would be advised to not only treat symptoms of the illness, but also to attend to perceptions of quality of life in the emotional domain. The difficulties that we observe here may underestimate limitations observed in actual clinical practice where individuals are more likely to have active disease state.

In an effort to understand why age and disease status exert an interactive effect on aspects of cognitive skills and HRQOL, we investigated two clinical variables in post hoc fashion that might serve as proxies for age by disease interactions, including medication burden and years of illness in predicting performance across cognitive domains, after considering the impact of education (for cognitive variables only) and symptom severity. Surprisingly, only years of illness were positively associated with HRQOL for physical role and overall mental health functioning, and only in the older group. This finding might suggest that individuals who have lived with $\mathrm{BPD}$ for the longest time have the best quality of life ratings, suggesting that experience with the illness over time may increase resilience. None of the clinical variables measured significantly predicted cognitive performance for any of the cognitive variables. It is notable that there is likely a complex relationship between medication use and cognitive functioning. For example, Lithium can confer neuroprotective effects, but also has anticholinergic properties that can alter cognitive functioning (Gray and McEwen, 2013). The proxy variables that we used for quantifying the interactive effects of age and disease were largely not successful in predicting variation in cognitive performance and HRQOL ratings in either younger or older adults. Other variables, such as number of hospitalizations, number of psychotic episodes, and/or neurobiological measures, such as cortisol may be better proxy variables to use in future study of mechanisms of age by disease interactions.

This study has a few limitations that should be considered in the interpretation of its results. First, this is a cross-sectional study, and although we can make inferences about how cognitive skills change over time among adults with BPD, only longitudinal studies can answer this question definitively. Other sources of cognitive differences between younger and older groups might relate to cohort differences, changes in treatment approaches throughout the years, and so on. Second, we chose to examine two distinct age groups, 18-29 years and 50-65 years, in order to include adults at either end of the younger and middle-age groups, and also not to confound results with potential hormonal variations that can occur during peri-menopause in women. However, this approach did not allow us to make inferences about cognitive changes that might occur during early middle age, which might also be relevant, or their longitudinal course. Third, most of the patients were medicated, and although we attempted to investigate the impact of medication burden on outcome variables, we were underpowered to more explicitly investigate specific medication effects. Fourth, it is possible that older patients with BPD experience a greater number of medical comorbidities than do other groups (Lala and Sajatovic, 2012), although this was not specifically assessed. Fifth, in exploratory regression analyses, we had to approach the impact of duration of illness on cognition and health-related quality of life ratings in a non-ideal manner because of the high correlation between age and duration of illness, disallowing for adequate disassociation of age-driven versus illness-loading effects. This would require a prospective longitudinal approach to address this question with any certainty. Finally, the sample was well educated. Given that education has been related to cognitive reserve (Liu et al., 2013), even greater differences in cognition among aging BPD patients may be detected in studies with less educated participants.

\section{Conclusion}

Aging in the context of BPD impacts specific cognitive skills, including aspects of executive functioning, processing speed, and emotion processing, as well as HRQOL in the physical role domain. Clinical variables that might help us to understand age by disease interactions in BPD were, by and large, not successful in predicting cognitive performance or HRQOL ratings. Future work might include the longitudinal assessment of euthymic BPD patients across the age spectrum with comprehensive neuropsychological evaluation and quality of life measurement, all of which 
are relevant to clinicians working with BPD patients. Knowing the progression of cognitive difficulties in aging BPD patients is likely to stimulate more specific cognitive rehabilitation trials that could function to help older BPD patients compensate for cognitive loss.

\section{Conflict of interest}

M. G. M. is on the Speakers Bureau for Merck Pharmaceuticals.

\section{Key Points}

- Late middle-aged euthymic adults with Bipolar Disorder (BPD) demonstrate particularly poor performance in the domains of emotion processing, processing speed, and aspects of executive functioning, relative to younger patients with BPD and same-age healthy peers.

- Verbal memory and other aspects of executive functioning, such as inhibitory control, conceptual reasoning, and set shifting are impacted only by age, regardless of disease status, while both aging and disease impact fine motor and visual memory skills, albeit independently.

- There is a double burden of aging and disease in BPD that impacts the reported ability of a person to perform daily physical tasks.

\section{Acknowledgements}

This research was supported by the Heinz C. Prechter Bipolar Research Fund at the University of Michigan Depression Center (M.G. M., D. M., A. L.W., K. R., A. V., M. K., S. A. L.). We also thank Ciaran Considine, Brennan Haase, Katie Hazlet, Nadia Huq, Lindsay Franti, Alison Kade, Michelle Kassel, Rachel Kay, Kortni Meyers, E. Michelle McFadden, Rachel Ringrose, Sarah Greenberg, Lauren Grove, Nicole Greer, and the rest of the staff of the Prechter Bipolar Research team for their contributions to this project. We thank an anonymous reviewer for suggestions that led to the creation of Figure 1 to better illustrate the complex interplay and potential interactions between age and disease. This work was presented in part at the 2012 Annual Meeting of the International Neuropsychological Society.

\section{References}

Almeida JR, Akkai D, Hassel S, et al. 2009. Reduced gray matter volume in ventral prefrontal cortex but not amygdala in bipolar disorder: significant effects of gender and trait anxiety. Psychiatry Res 171: 54-68.

Altshuler LL, Ventura J, van Gorp WG, et al. 2004. Neurocognitive function in clinically stable men with bipolar I disorder or schizophrenia and normal control subjects. Biol Psychiatry 56: 560-569.

Bonnin CM, Sanchez-Moreno J, Martinez-Aran A, et al. 2012. Subthreshold symptoms in bipolar disorder: impact on neurocognition, quality of life and disability. J Affect Disord 136: 650-659.

Bora E, Yucel M, Pantelis C. 2009. Cognitive endophenotypes of bipolar disorder: a meta-analysis of neuropsychological deficits in euthymic patients and their firstdegree relatives. J Affect Disord 113: 1-20.

Braw Y, Erez G, Sela T, et al. 2013. A longitudinal study of cognition in asymptomatic and mildly symptomatic bipolar disorder patients. Psychiatry Res 210: 842-849.

Burdick KE, Goldberg JF, Harrow M, et al. 2006. Neurocognition as a stable endophenotype in bipolar disorder and schizophrenia. J Nerv Ment Dis 194: $255-260$.

Delaloye C, Moy G, Baudois S. 2009. Cognitive features in euthymic bipolar patients in old age. Bipolar Disord 11: 735-743.

Delaloye C, Moy G, de Bilbao F, et al. 2011. Longitudinal analysis of cognitive performances and structural brain changes in late-life bipolar disorder. Int J Geriatr Psychiatry 26: 1309-1318.

Depp CA, Savia GN, Moore DJ, et al. 2008. Short-term course of neuropsychological abilities in middle-aged and older adults with bipolar disorder. Bipolar Disord 10: 684-690.

Gildengers AG, Chisholm D, Butters MA, et al. 2012. Two-year course of cognitive function and instrumental activities of daily living in older adults with bipolar disorder: evidence for neuroprogression? Psychol Med 43: 1-11.

van Gorp WG, Altshuler L, Theberge DC, et al. 1998. Cognitive impairment in euthymic bipolar patients with and without prior alcohol dependence. a preliminary study. Arch Gen Psychiatry 55: 41-46.

Gray JD, McEwen BS. 2013. Lithium's role in neural plasticity and its implications for mood disorders. Acta Psychiatr Scand 128: 347-361.

Gualtieri CT, Johnson LG. 2008. Age-related cognitive decline in patients with mood disorders. Prog Neuropsychopharmacol Biol Psychiatry 32: 962-967.

Hamilton M. Development of a rating scale for primary depressive illness. 1967. Br J Soc Clin Psychol 6: 278-296.

Hassel S, Almeida JR, Kerr N, et al. 2008. Elevated striatal and decreased dorsolateral prefrontal cortical activity in response to emotional stimuli in euthymic bipolar disorder: no associations with psychotropic medication load. Bipolar Disord 10: 916-927.

Lala SV, Sajatovic M. 2012. Medical and psychiatric comorbidities among elderly individuals with bipolar disorder: a literature review. J Geriatr Psychiatry Neurol 25: 20-25.

Langenecker SA, Saunders EF, Kade AM, et al. 2010. Intermediate: cognitive phenotypes in bipolar disorder. J Affect Disord 122: 285-293.

Liu Y, Cai ZL, Xue S, et al. 2013. Proxies of cognitive reserve and their effects on neuropsychological performance in patients with mild cognitive impairment. J Clin Neurosci 20: 548-553.

Martinez-Aran A, Vieta E, Colom F, et al. 2004. Cognitive impairment in euthymic bipolar patients: implications for clinical and functional outcome. Bipolar Disord 6: 224-232.

McHorney C, Ware J, Raczek A. 1993. The MOS 36-Item Short-Form Health Survey (SF-36): II. Psychometric and clinical tests of validity in measuring physical and mental health constructs. Med Care 31: 247-263.

McHorney C, Ware JE, Lu JF, et al. 1994. The MOS 36-item Short-Form Health Survey (SF-36): III. Tests of data quality, scaling assumptions, and reliability across diverse patient groups. Med Care 32: 40-66.

McKinney BC, Sibille E. 2013. The age-by-disease interaction hypothesis of late-life depression. Am J Geriatr Psychiatry 21: 418-432.

Michalak EE, Yatham LN, Lam RW. 2005. Quality of life in bipolar disorder: a review of the literature. Health Qual Life Outcomes 3: 72.

Moorhead TW, McKirdy J, Sussmann JE, et al. 2007. Progressive gray matter loss in patients with bipolar disorder. Biol Psychiatry 62: 894-900.

Nurnberger JI, Jr, Blehar MC, Kaufmann CA, et al. 1994. Diagnostic interview for genetic studies. Rationale, unique features, and training. NIMH Genetics Initiative. Arch Gen Psychiatry 51: 849-859.

Rubinsztein JS, Michael A, Paykel ES, et al. 2000. Cognitive impairment in remission in bipolar affective disorder. Psychol Med 30: 1025-1036.

Sarnicola A, Kempton M, Germana C, et al. 2009. No differential effect of age on brain matter volume and cognition in bipolar patients and healthy individuals. Bipolar Disord 11: 316-322.

Schouws SN, Comijs HC, Stek ML, et al. 2009. Cognitive impairment in early and late bipolar disorder. Am J Geriatr Psychiatry 17: 508-515.

Torrent C, Martinez-Aran A, del Mar Bonnin C, et al. 2012. Long-term outcome of cognitive impairment in bipolar disorder. J Clin Psychiatry 73: e899-e905. 
Torres IJ, Bodreau VG, Yatham LN. 2007. Neuropsychological functioning in euthymic bipolar disorder: a meta-analysis. Acta Psychiatr Scand 116(Suppl. 434): $17-26$.

Villardita C, Cultrera S, Cupone V, et al. 1985. Neuropsychological test performances and normal aging. Arch Gerontol Geriatr 4: 311-319.

Ware J, Sherbourne C. 1993. The MOS 36-item short-form health survey (SF-36). I. Conceptual framework and item selection. Med Care 30: 473-483.

Young RC, Biggs JT, Ziegler VE, et al. 1978. A rating scale for mania: reliability, validity and sensitivity. Br J Psychiatry 133: 429-435.
Zubieta JK, Huguelet P, O'Neil RL, et al. 2001. Cognitive function in euthymic bipolar I disorder. Psychiatry Res 102: 9-20.

\section{Supporting information}

Additional supporting information may be found on the online version of this article at the publisher's web site. 